that allows care professionals, volunteers and family carers to use music designed specifically for end of life care.

Aim To extend delivery and accessibility of benefit-designed music resources into residential and community hospice palliative care (HPC) sites across Canada; to gather information on how designed music resources impact quality of life and care in HPC.

Methods Recruited 84 HPC sites across Canada; pre-test survey; provision of music care kits with instruction, education and support to point person in each HPC; four month implementation and evaluation with support, post-test questionnaire and telephone interview; one-year follow up online survey, qualitative coding analysis.

Results

- predictors for developing music care programs in a Canadian HPC site

- five impact themes including improved quality of life, assisted relationship completion, decreased feelings of fear and isolation, helped to meet psychosocial and spiritual needs, and provided distraction

- production of a controlled delivery and support process for music care

- caregiver feedback

- 'having the music playing in the room was a wonderful distraction and reduced his calling out and restlessness.'

- 'When we gave him the music, it was the first time he slept in the 36 hours.'

- 'When we used the music, the patient closed her eyes and immediately was calmer.'

Conclusions R2R created opportunities for Canadian HPC sites to offer music care to families without the need of a music therapist, facilitated by the provision of designed resources at no cost to $\mathrm{R} 2 \mathrm{R}$ participants. Results show that music care improved the experience of patient, loved ones and care providers at end of life.

\section{P-68 SINGING TO A DEAD INFANT: HOW MUSIC THERAPY PROMOTED AND VALUED CULTURAL DIFFERENCE}

Victoria Swan. Demelza, Hospice Care for Children, Sittingbourne, UK

\subsection{6/bmispcare-2017-hospice.95}

Background It has long been acknowledged that death is beyond a biological phenomenon but a social and cultural one, which is understood within complex cultural meanings. This presentation outlines music therapy support, delivered to a Yoruba infant and his mother, through end of life care and post death.

Methods In the Yoruba culture, death is not perceived as the end of life, but a transition through existence. Therefore it was a priority and responsibility to respect and adapt to the mother's emotional, spiritual and cultural beliefs. This presentation will discuss how a Music Therapist valued and responded to the cultural beliefs of a Yoruba mother through end of life care for her baby, and subsequently by singing to her deceased baby, providing the mother with reassurance through feedback. The issues encountered as a practitioner around culture will be explored during the presentation.

Results This grieving mother desperately wanted her child's next life to be a healthy one. In her belief, the music therapy made this happen. The mother's response to this intervention was 'You don't know what this means to me.' Although cultural knowledge is a key component to culturally competent intervention, skills of communication rather than mastery of cultural traits, underpinned the therapeutic support. A recommendation for professionals working in palliative care will be made to take a broader view around cultural elements of death, with the emphasis being on skills such as communication.

Conclusions The playing of music was viewed as vital to guide the baby's soul to find his next life. This work challenges the notion of cultural competency in therapeutic intervention within palliative care. Skilful and compassionate communication with the family enabled the Music Therapist to value, promote and support cultural difference.

\section{P-69 MUSIC CARE TRAINING PROGRAM: INTEGRATING MUSIC INTO REGULAR CARE PRACTICE}

Bev Foster. Room 217 Care through Music, Toronto, Canada

\subsection{6/bmjspcare-2017-hospice.96}

Background In Canada, music is identified as a non-pharmacological, cost-effective and person-centred approach across the care spectrum, including palliative care. Caregiver confidence and music medicine knowledge translation are barriers to its use. Music Care Training (MCT) is an education program assisting care partners in the effective and responsible use of music in care.

Aim Provide musical tools and theoretical context to enrich care practice; Develop understanding of music care approach, musical elements and evidence base for using music in care; creating music care initiative for care context.

Methods Hold music care trainings in identified Canadian city-centres; pre-test questionnaire, training in on-site classes of maximum 30, post-test questionnaire, three month online follow up, measure social return on investment (SROI).

Results

- 800 care partners trained in 36 months, 19\% hospice palliative care context, 56\% long term care context (advanced dementia care).

- Level 1 - caregiver confidence increased in ability to use music care kit, design music care plan and explain music as an approach to care.

- Level 2 - caregiver confidence increased to describe music's effects on whole person care, including neuroplasticity, pain management, mood, memory and meaning-making.

- Level 3 - demonstrated music care initiative implemented in $86 \%$ of participants.

- $95.51 \%$ student satisfaction level.

- Key performance indicators developed to measure SROI. Caregivers Stories 'I have used the training several times. One resident came out of a showering experience sobbing, shivering, rocking, crying and moaning shock. I was able to use the call and response, the humming, the singing and the breathing to calm her and lull her to sleep. Without your training I may have been at a loss as I have never seen this resident so upset.'

Conclusions Music Care Training is giving care partners in Canada the knowledge and confidence needed to use music care. It is a replicable model for the UK. 